\title{
Efficient Generation of Non-Integration and Feeder-Free Induced Pluripotent Stem Cells from Human Peripheral Blood Cells by Sendai Virus
}

\author{
Huahu Ye Qiwei Wang \\ Cell Engineering Laboratory, Beijing Institute of Biotechnology, 20 Dongda Street, Fengtai District, \\ Beijing, China
}

\section{Key Words}

Induced pluripotent stem cells - Sendai virus - Reprogramming - Extracellular substrates • Feeder-free • Rock inhibitors

\begin{abstract}
Background/Aims: Induced pluripotent stem cells (iPSCs) hold great promise for regenerative medicine, disease modeling, and drug development. Thus, generation of non-integration and feeder-free iPSCs is highly desirable for clinical applications. Peripheral blood mononuclear cells (PBMCs) are an attractive resource for cell reprogramming because of their properties of easy accessibility and the limited invasiveness of blood collection. However, derivation of iPSCs is technically demanding due to the low reprogramming efficiency and nonadherent features of PBMCs. Methods: iPSCs were generated from PBMCs using non-integrative Sendai viruses carrying the reprogramming factors Oct4, Sox2, KIf4, and CMyc. The derived iPSCs were fully characterized at the levels of gene and protein, and then they were transplanted into immunocompromised mice for evaluation of in vivo differentiation potential. Three types of extracellular substrates (Geltrex, vitronectin, and rhLaminin-521) were tested for their influences on cell reprogramming under feeder-free conditions. We also sought to establish approaches to efficient cell recovery post-thaw and single cell passaging of iPSCs employing Rock inhibitors. Results: iPSCs were efficiently generated from PBMCs under feeder-free conditions. The derived iPSCs proved to be pluripotent and transgene-free. Furthermore, they demonstrated multi-lineage differentiation potentials when transplanted into immunocompromised mice. Among the three substrates, Geltrex and rhLaminin-521 could effectively support the initial cell reprogramming process, but vitronectin failed. However, the vitronectin, similar to Geltrex and rhLaminin-521, could effectively maintain cell growth and expansion of passaged iPSCs. In addition, RevitaCell supplement (RVC) was more potent on cell recovery post-thaw than Y-27632. And RVC and Y-27632 could significantly increase the cell survival when the cells were passaged in single cells, and they showed comparable effectiveness on cell recovery. Conclusion: We have successfully derived non-integration and feeder-free human iPSCs from




\section{Cellular Physiology Cell Physiol Biochem 2018;50:1318-1331 \\ \begin{tabular}{ll|l} 
and Biochemistry Published onlIne: 25 October 2018 & $\begin{array}{l}\text { (c) } 2018 \text { The Author(s). Published by S. Karger AG, Basel } \\
\text { www.karger.com/cpb }\end{array}$ \\
\hline
\end{tabular} \\ Ye et al.: Peripheral Blood Cells-Derived Non-Integration and Feeder-Free iPSCs}

peripheral blood cells, and established effective strategies for efficient cell recovery and single cell passaging. This study will pave the way to the derivation of clinical-grade human iPSCs for future clinical applications.

(C) 2018 The Author(s)

Published by S. Karger AG, Basel

\section{Introduction}

Induced pluripotent stem cells (iPSCs) were first established from mouse fibroblasts by Yamanaka in 2006 [1]. Subsequently, human iPSCs were successfully derived by the Yamanaka and Thomson groups [2,3]. By ectopic-expression of a defined set of reprogramming factors (Oct4, Sox2, Klf4, and cMyc/Oct4, Sox2, Nanog, and Lin28), iPSCs have been generated from many cell types of different species [1-8]. Retrovirus or lentivirus was initially employed to deliver reprogramming factors for iPSC generation $[1,3]$. These genome-integrating methods could potentially produce tumorigenic insertional mutations, and residual or reactivation of transgene expression during iPSC differentiation could influence their in vitro differential capacity and the functionality of iPSC derivatives $[3,9]$. To solve these problems, various methods were explored to derive transgene-free iPSCs, such as plasmid vectors, minicircle DNA vectors, piggyBac, mRNA, adenovirus, Sendai virus, proteins, and small molecules [1017].

Transient expression of reprogramming factors by episomal plasmids would allow for the derivation of transgene-free iPSCs. However, transient transfection with a standard plasmid vector cannot provide a long enough expression period of time for cell reprogramming [14]. OriP/EBNA-1 (Epstein-Barr nuclear antigen-1)-based plasmids are stably expressed for a longer period of time than standard plasmids, and higher transfection efficiency can be achieved with these vectors due to the oriP/EBNA-1-mediated nuclear import and retention of vector DNA [18]. A single transfection of episomal plasmid vectors is sufficient for the derivation of iPSCs.

Expression of reprogramming factors using mRNA provides another method to make transgene-free iPSCs. It was shown that in vitro transcribed mRNAs were able to efficiently express reprogramming factors when transfected into human fibroblasts [19]. Although reprogramming factor mRNAs are commercially available, this method suffers from the limitations that it is labor-intensive, requires daily transfection of mRNA for 7 successive days, and there are no successful reports regarding the reprogramming of blood cells.

Sendai virus is a single chain RNA virus that does not integrate into the host genome or alter the genetic information of the host cells. The virus remains in the cytoplasm and is therefore diluted out of the host cells $\sim 10$ passages after virus infection. Sendai virus can infect a wide range of cell types in proliferative and quiescent states with high transduction efficiency. Expression of transgenes delivered by Sendai virus is detectable as early as 6-10 hrs after transduction, with maximum expression detected more than $24 \mathrm{hrs}$ after transduction. Sendai-based reprogramming vectors have been used to successfully reprogram neonatal and adult fibroblasts as well as blood cells with high efficiency [10, 20,21].

Overall, episomal vectors, mRNA, and Sendai virus are the most often used nonintegrating methods for iPSCs generation among the aforementioned methods. However, mRNA has a limited applicability to somatic cell types, and there is not yet any report demonstrating successful reprogramming of blood cells. Episomal vectors have relatively low reprogramming efficiencies compared to the Sendai virus method. In this report, we successfully generated non-integration and feeder-free iPSCs from human peripheral blood cells by Sendai virus. We tested three different types of substrates (Geltrex, vitronectin, and rhLaminin-521) for their influences on cell reprogramming. Among these substrates, Geltrex and rhLaminin-521 could effectively support the initial cell reprogramming process, and the passaged iPSCs could grow and expand well on all three substrates. We also compared the effects of two Rock inhibitors, RevitaCell supplement (RVC) and Y-27632 on cell recovery after cell thawing and demonstrated that RVC was more effective on cell recovery than Y-27632. Furthermore, RVC and Y-27632 showed comparable effectiveness on single cell 


\section{Cellular Physiology Cell Physiol Biochem 2018;50:1318-1331 \begin{tabular}{ll|l} 
DOl: 10.1159/000494589 & $\begin{array}{l}\text { O } 2018 \text { The Author(s). Published by S. Karger AG, Basel } \\
\text { www.karger.com/cpb }\end{array}$
\end{tabular} \\ Ye et al.: Peripheral Blood Cells-Derived Non-Integration and Feeder-Free iPSCs}

passaging, and they all provided great improvements in cell survival and recovery of iPSCs. Together, the current study demonstrates that non-integration and feeder-free iPSCs can be effectively derived from human peripheral blood cells by Sendai virus. Considering safety issues, derivation of footprint-free and xeno-free iPSCs is highly important for their future clinical applications.

\section{Materials and Methods}

Ethical approval

Blood samples were collected from five healthy voluntary donors, ranging from 32 to 53 years old, with written consent permitted by the ethical committee of the Beijing Institute of Biotechnology.

Isolation and culture of peripheral blood mononuclear cells (PBMCS)

PBMCs were isolated by density gradient centrifugation with Ficoll-paque Plus ( $p=1.077 \pm 0.001 \mathrm{~g}$ ) $\mathrm{ml}$ (GE Healthcare,17-1440-02) from $5 \mathrm{ml}$ whole blood according to the manufacturer's instructions and cryopreserved in the liquid nitrogen tank for future application. Four days before transduction, frozen PBMCs were quickly thawed and cultured in complete PBMC medium containing complete StemPro-34 SFM medium supplemented with $100 \mathrm{ng} / \mathrm{ml}$ stem cell factor (SCF, Life Technologies, PHC2111), $100 \mathrm{ng} / \mathrm{ml}$ FLT3 (Life Technologies, PHC9414), $20 \mathrm{ng} / \mathrm{ml}$ interleukin-3 (IL3, Life Technologies, PHC0034), and $20 \mathrm{ng} /$ $\mathrm{ml}$ interleukin-6 (IL6, Life Technologies, PHC0065). Medium was half replaced with fresh complete PBMC medium everyday for 3 days (Day -3 to Day -1). Complete StemPro-34 SFM medium consisted of StemPro-34 SFM medium (1X) (Life Technologies, 10639-011) supplemented with StemPro-34 Nutrient Supplement and $2 \mathrm{mM} \mathrm{L-Glutamine} \mathrm{(Life} \mathrm{Technologies,} \mathrm{25030-081).}$

\section{Generation of iPSCs from PBMCs with Sendai virus}

The scheme of generating iPSCs is shown in Fig. 1A. The process was as follows. After 4 days cell culture (Day 0), the cells were collected and the number of cells were counted. $3 \times 10^{5}$ live cells were transduced by the Klf4, Oct4, and Sox2 (KOS) virus, and the c-Myc virus at a multiplicity of infection (MOI) of 5 (KOS MOI=5, hc-Myc MOI=5), and the Klf4 virus at an MOI of 3 (hKlf4 MOI=3) (CytoTune®-iPSC2.0 Sendai Reprogramming Kit, Life Technologies, A16517) in a total volume of $1 \mathrm{ml}$. The transduction was performed in complete PBMC medium with $4 \mu \mathrm{g} / \mathrm{ml}$ of Polybrene by centrifugation at 2250 RPM for $30 \mathrm{~min}$ at room temperature. And then the pellet was resuspended in the same medium containing the virus and plated in a 24-well plate and incubated overnight at $37^{\circ} \mathrm{C}$. The day after transduction (Day 1), Sendai viruses were removed by centrifuging the cell suspension. The cells were resuspended in a 24-well plate with fresh complete PBMC medium for 2 days. At day 3, the cells were collected by centrifugation, resuspended with complete StemPro-34 SFM medium without cytokines, and plated onto Geltrex-, vitronectin-, and rhLaminin521-coated 6-well plates (Life Technologies, A1413301, A14700, and A29248). The medium was refreshed every other day. At day 7, transitioning into iPSC medium began by replacing half of the StemPro-34 SFM medium without cytokines with Essential 8 (E8) medium (Life Technologies, A151700). From day 8 on, the full volume of medium was changed to E8 medium. The medium was replaced everyday thereafter and the emergence of iPSC colonies was monitored. Around day 15 to 21 after transduction, colonies were ready for transfer. The colonies were manually picked and transferred onto different substrate-coated 12 -well plates for expansion.

\section{RNA isolation and reverse transcription}

Total RNA was purified with TRIzol reagent (Life Technologies, 15596026) and digested with DNase to remove genomic DNA contamination. $1 \mu \mathrm{g}$ of total RNA was used for reverse transcription reaction with Superscript IV first-strand synthesis system (Life Technologies, 18091050), according to the manufacturer's instructions. Polymerase chain reaction (PCR) was performed with Taq DNA polymerase (Life Technologies, 10342053). The PCR conditions were as follows: initial denaturation at $94^{\circ} \mathrm{C}$ for $3 \mathrm{~min}$ followed by 30 cycles of denaturation at $94^{\circ} \mathrm{C}$ for $30 \mathrm{~s}$, annealing at $56^{\circ} \mathrm{C}$ for $30 \mathrm{~s}$, extension at $72^{\circ} \mathrm{C}$ for $1 \mathrm{~min}$, and a final extension at $72^{\circ} \mathrm{C}$ for $10 \mathrm{~min}$. Primer information is shown in Table 1.

\section{KARGER}




\section{Cellular Physiology Cell Physiol Biochem 2018;50:1318-1331 \begin{tabular}{l|l|l} 
and Biochemistry & DOI: 10.1159/000494589 & $\begin{array}{l}\text { C } 2018 \text { The Author(s). Published by S. Karger AG, Basel } \\
\text { www.karger.com/cpb }\end{array}$
\end{tabular}

Table 1. Primers for RT-PCR

\begin{tabular}{lcccccc}
\hline Genes & Accession & Forward & Reverse & Size & Tm & Cycles \\
\hline Oct4 & NM_002701 & tctcccatgcattcaaactg & gtttcttccctagctcctc & 80 & 56 & 30 \\
Sox2 & NM_003106 & cccggcacggccattaac & acccctcccatttccctc & 109 & 56 & 30 \\
Nanog & NM_024865 & cctgtgatttgtgggcctg & gacagtctccgtgtgaggcat & 78 & 56 & 30 \\
Klf4 & NM_001314052 & gggttcaggtgccccagc & tgtcagactcgccaggtg & 85 & 56 & 30 \\
cMyc & NM_002467 & ctgccaggacccgcttct & gtttccactacccgaaa & 76 & 56 & 30 \\
GAPDH & NM_002046 & cgagatccctccaaaatcaagt & tgaggctgttgtcatacttctcat & 196 & 56 & 30 \\
Sev & AB855655 & ggatcactaggtgatatcgagc & accagacaagagtttaagagatatgtatc & 181 & 56 & 30 \\
\hline
\end{tabular}

Flow cytometry analysis

Cells were analyzed for Oct4, Sox2, and SSEA-4, using Human/Mouse Pluripotent Stem Cell Multi-Color Flow Cytometry Kit (R\&D Systems, FMC001) according to the manufacturer's instructions. The corresponding isotype-matched antibodies were used as negative controls. Cells $(20,000$ events per sample) were acquired on a FACS Calibur flow cytometer (BD Biosciences) and data were analyzed using FCS express 4 plus software.

Alkaline phosphatase (AP) staining, karyotyping, and immunofluorescence (IF) analysis

After 3 passages, cells were subjected to AP staining according to the manufacturer's protocol using the AP Detection Kit (Stemgent, 00-0055) for pluripotency detection. Briefly, cells were fixed in Fix Solution for 2-5 min at room temperature. Then cells were incubated in freshly prepared AP Substrate Solution for 10-20 min in the dark. Authentic iPSC colonies were stained positive for alkaline phosphatase, displaying a red or purple stain. For karyotype analysis, cell division was blocked in mitotic metaphase using colcemid-spindle formation inhibitor (Invitrogen, 15212). G-band standard staining (Giemsa, Invitrogen, 10092013) was used for the chromosome visualization. For the detection of pluripotency markers by IF, cells were grown in 6-well plates and fixed with $4 \%$ paraformaldehyde for $30 \mathrm{~min}$ at $4{ }^{\circ} \mathrm{C}$. After that, cells were permeabilized and blocked with $10 \%$ calf serum and $0.1 \%$ Triton X-100 in PBS. Subsequently, cells were stained with primary antibodies overnight at $4{ }^{\circ} \mathrm{C}$ and finally incubated with fluorochrome-labeled secondary antibodies. The primary antibodies used for detecting pluripotency markers were anti-Oct4 (Santa Cruz, sc-5279), antiSox2 (Calbiochem, sc1002), anti-Nanog (Santa Cruz, sc-33759), and anti-SSEA-4 (Santa Cruz, sc-59368). The secondary antibodies used were donkey anti-mouse AF488, donkey anti-mouse AF594, and donkey antirabbit AF594 (Life Technologies, A21202, A21203, and A21207). Nuclei were stained by Hoechst 33258 (Sigma Aldrich, 94403). Negative staining controls were carried out by replacing the primary antibody with rabbit or mouse sera. Images were captured using Nikon Eclipse Ti-U microscope, and images were processed using Adobe Photoshop CS5.

\section{Bisulfite sequencing}

Genomic DNA was isolated from the cells and modified with sodium bisulfite using the Epitect Bisulfite Kit (Qiagen, 59104) according to the manufacturer's instructions. Methylation-specific PCR primers are shown in Table 2. Amplified products were cloned into pGEM®-T Vector System (Promega, A3600). Randomly selected clones were sequenced with the T7 forward primer for the gene and analyzed for methylation changes at CpG sites of the Oct4 promoter $(-662$ to +25 , from transcriptional start site (TSS)).

\section{Teratoma formation}

Cells were harvested by $0.5 \mathrm{mM}$ EDTA treatment, collected into conical tubes, and centrifuged, and the pellets were resuspended in E8 medium. 3 X $10^{6}$ cells/mouse were injected subcutaneously into the dorsal flank of immunocompromised mice ( $n=6$ /group). Around 12 weeks, mice were euthanized when a visible tumor mass was detected. The teratomas were dissected and fixed in $10 \%$ formalin. Samples were 


\section{Cellular Physiology Cell Physiol Biochem 2018;50:1318-1331 \begin{tabular}{ll|l} 
DOI: 10.1159/000494589 & $\begin{array}{l}\text { O 2018 The Author(s). Published by S. Karger AG, Basel } \\
\text { www.karger.com/cpb }\end{array}$
\end{tabular}}

embedded in paraffin and processed with hematoxylin and eosin staining. The teratomas were confirmed by histological analysis. The remaining mice, without visible tumors, were euthanized at week 16 . All mice were examined for teratoma formation by gross and microscopic inspection. All animal procedures were approved by the Institutional Animal Care and Use Committee (IACUC) of the Beijing Institute of Biotechnology.

\section{Freezing and thawing of iPSCs}

When cells reached a confluency of $70-80 \%$, the spent medium was aspirated out and the cells were treated with $0.5 \mathrm{mM}$ EDTA. Then the cells were incubated at $37^{\circ} \mathrm{C}$ for $3-5 \mathrm{~min}$ and gently transferred into conical tubes, leaving the cells in clumps. The cell suspension was centrifuged and the pellet was resuspended in freezing medium containing E8 medium supplemented with 10\% DMSO. The cell suspension was aliquoted into cryovials and placed in a cryofreezing container at $-80^{\circ} \mathrm{C}$. After overnight storage at $-80^{\circ} \mathrm{C}$, the cells were transferred to a liquid nitrogen tank for long-term storage. For thawing and recovering cells, the cryovial was immersed in a $37^{\circ} \mathrm{C}$ water bath for $1-2 \mathrm{~min}$. Then the cells were gently transferred to a $15 \mathrm{ml}$ conical tube containing pre-warmed E8 medium. The cells were centrifuged and the pellet was resuspended in $2 \mathrm{ml}$ of E8 medium supplemented with either 1X RVC (Life Technologies, A2644501) or 10 $\mu \mathrm{M}$ Y-27632 (Calbiochem, 688000). The cells were cultured on vitronectin-coated 6-well plates. The next day, the cells were refreshed with fresh E8 medium. The medium was replaced daily thereafter until the cells are approximately $80 \%$ confluent. To determine the efficiency of the cell recovery following cell thawing, images were captured by phase-contrast microscopy at regular intervals. For measuring the proliferation of cells after cell thawing, cell masks were calculated by NIS-Elements AR analysis (Nikon) to determine the percentage change in confluency of the cells over time.

\section{Kinetic growth of single iPSCs}

For single cell passaging, cells were rinsed with DPBS and treated with TrypLE ${ }^{\mathrm{TM}}$ Express Enzyme (Life Technologies, 12604-013) for $5 \mathrm{~min}$. Next, the cells were triturated several times to efficiently singularize the cells. Finally, the cells were centrifuged and resuspended, and cultured $\left(10,000\right.$ cells $\left./ \mathrm{cm}^{2}\right)$ in E8 medium supplemented with either 1X RVC or $10 \mu \mathrm{M}$ Y-27632 for 24 hrs post-split, followed by daily feeding with E8 medium alone for the remainder of the culture. To determine the kinetic growth of the cells following single cell passaging, images were captured by phase-contrast microscopy at regular intervals, and cell masks were calculated to determine the proliferation of cells over time.

\section{Statistical analysis}

All the experiments were carried out at least three times. Data were displayed as mean \pm SD. To assess statistical significance, two-tailed, unpaired Student's $t$ test was performed and $p<0.05$ was considered significant.

\section{Results}

\section{iPSC generation from peripheral blood}

PBMCs were isolated from $5 \mathrm{ml}$ whole blood of healthy donors by density gradient centrifugation, and the cells were cryopreserved for the following iPSC generation. In general, 1 X $10^{6}$ PBMCs could be efficiently purified from $1 \mathrm{ml}$ whole blood and that is sufficient for iPSC derivation. Four days before cell reprogramming, the frozen PBMCs were quickly thawed and cultured in complete PBMC medium with cytokines SCF, FLT3, IL3, and IL6 (Fig. 1A). After 4 days recovery, $3 \times 10^{5}$ live cells were transduced by the Klf4, Oct4, Sox2, and c-Myc viruses with the manufacturer recommended MOI. To increase the transduction and reprogramming efficiencies, the transduction was performed by centrifugation at 2250 RPM for $30 \mathrm{~min}$ at room temperature, and this greatly increased the number of colonies. For derivation of feeder-free iPSCs, we employed extracellular substrates to replace feeder cells for iPSC generation. Three types of substrates were evaluated for supporting cell reprogramming. At day 3 after transduction, the cells were replated onto Geltrex (1:100)-, vitronectin $\left(0.5 \mu \mathrm{g} / \mathrm{cm}^{2}\right)$-, and rhLaminin-521 $\left(0.5 \mu \mathrm{g} / \mathrm{cm}^{2}\right)$-coated 6-well plates, respectively. 


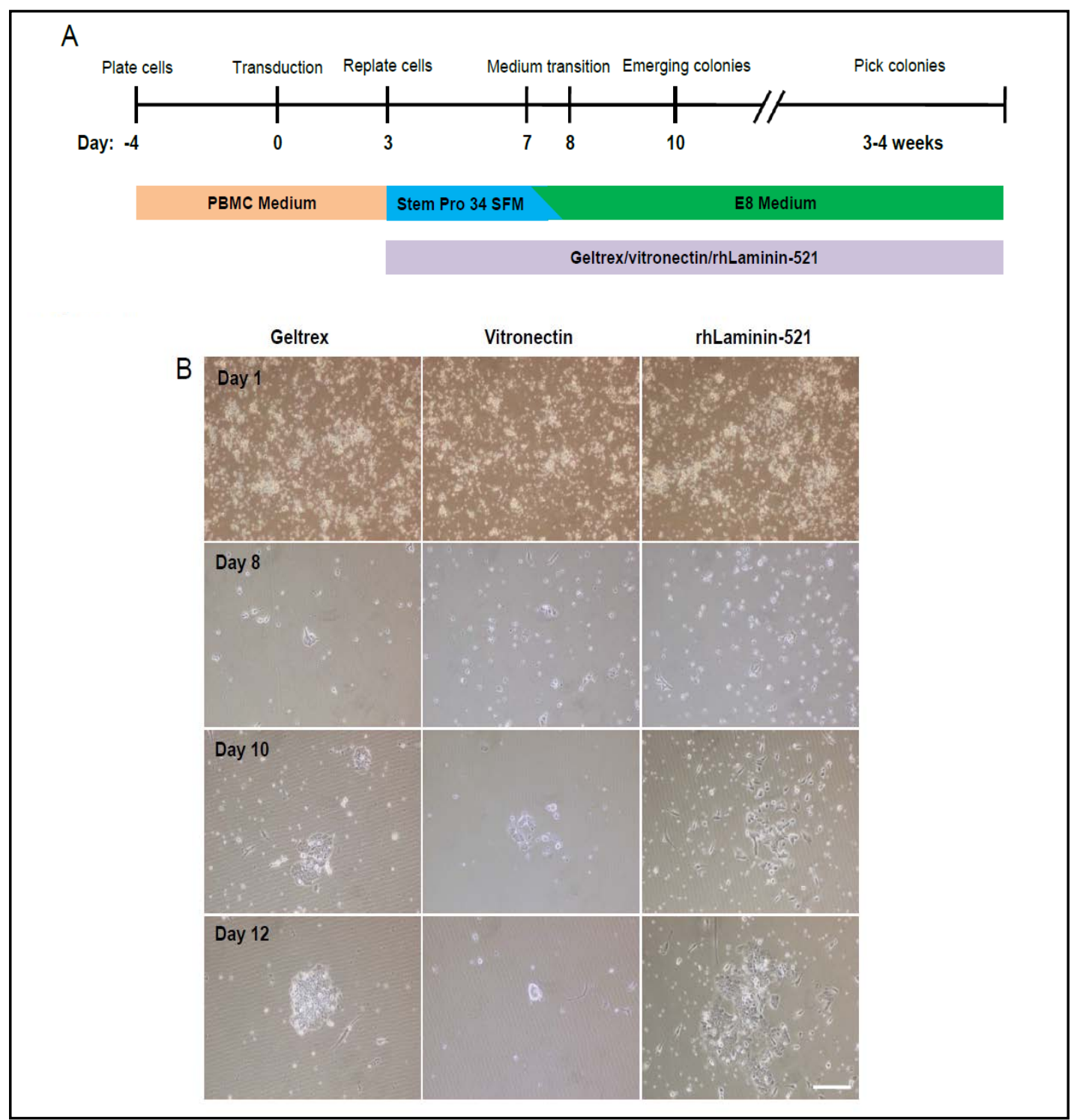

Fig. 1. Generation of human iPSCs from PBMCs by Sendai virus. A. Schematic diagram of derivation of iPSCs. After 4 days cell culture, PBMCs were transduced by Sendai viruses at MOI=5-5-3 (KOS MOI=5, hc-Myc $\mathrm{MOI}=5$, and hKlf4 MOI=3). One day after transduction, Sendai viruses were removed and the cells were cultured in complete PBMC medium for 2 days. At day 3, the cells were replated onto Geltrex-, vitronectin-, and rhLaminin-521-coated plates, respectively. At day 7, transitioning into E8 medium began. Around day 15 to 21 after transduction, colonies were manually picked and transferred onto different substratecoated plates for expansion and characterization. B. Effects of extracellular substrates on the initial cell reprogramming stage. About 1 week after transduction, some small colonies began emerging on all three types of substrate-coated plates. The number of colonies on vitronectin substrate was less than those on Geltrex and rhLaminin-521 substrates, respectively. The numbers of colonies on Geltrex and rhLaminin-521 substrates were comparable. The colonies on vitronectin substrate were easy to detach from the plates when changing the medium. Bar is $200 \mu \mathrm{m}$.

Around 1 week after transduction, some small colonies began emerging on all three types of substrate-coated plates. However, the number of colonies on vitronectin substrate was less than those on Geltrex and rhLaminin-521 substrates, respectively. The numbers of colonies on Geltrex and rhLaminin-521 substrates were comparable. Furthermore, the colonies on vitronectin substrate were not firmly attached to the plates during the reprogramming, 


\section{Cellular Physiology Cell Physiol Biochem 2018;50:1318-1331 \\ \begin{tabular}{ll|l} 
DOl: 10.1159/000494589 & $\begin{array}{l}\text { O } 2018 \text { The Author(s). Published by S. Karger AG, Basel } \\
\text { www.karger.com/cpb }\end{array}$ \\
\cline { 2 - 4 }
\end{tabular} \\ Ye et al.: Peripheral Blood Cells-Derived Non-Integration and Feeder-Free iPSCs}

and easy to detach from the plates when changing the medium (Fig. 1B). Even though we increased the concentration of vitronectin to $1 \mu \mathrm{g} / \mathrm{cm}^{2}$, there was not much difference on reprogramming compared to using $0.5 \mu \mathrm{g} / \mathrm{cm}^{2}$. Therefore, it might be difficult to successfully reprogram PBMCs on vitronectin substrate due to loosing attachment between the colonies and vitronectin substrate during reprogramming. Around 2-3 weeks, the size of the colonies was suitable for picking. The flat and compact colonies with clear boundaries were selected for transfer. In our experiments, around 30 human embryonic stem cell (ESC)-like colonies per 3 X $10^{5}$ PBMCs were obtained (33 colonies for Geltrex-iPSCs and 29 colonies for rhLaminin521-iPSCs, respectively). The reprogramming efficiency was approximately $0.01 \%$ (The efficiency was calculated as the number of emerging human ESC-like colonies per starting cell number). Although the vitronectin substrate was inefficient to support the initial cell reprogramming process, the passaged colonies could successfully grow and expand on this substrate. There was not much difference observed on cell growth and expansion during the cell culturing on the three types of substrate (Geltrex, vitronectin and rhLaminin-521)coated plates. In addition, we also successfully derived iPSCs from the other four donors' PBMCs with similar reprogramming efficiencies and timing.

\section{Characterization of iPSCs}

When picked and expanded in E8 medium, iPSCs showed typical human ESC morphology, i.e. flat and compact colonies with clear boundaries, high nucleus/cytoplasm ratios and prominent nucleoli, and they robustly proliferated for more than 20 passages and maintained normal karyotypes (Fig. 2A). These iPSCs were positive for AP staining (Fig. 2A). To test pluripotency gene expression, total RNA was extracted from iPSCs. The results showed that iPSCs expressed pluripotency markers Oct4, Sox2, Nanog, Klf4, and cMyc (Fig. 2B). RT-PCR analysis also indicated that Sendai virus was retained in these iPSCs up to passage 10, which is consistent with the previous report [22]. To obtain virus-free iPSCs, we performed a temperature shift to remove the virus. After incubating the cells at $38^{\circ} \mathrm{C}$ for 5 days, Sendai virus was completely absent in passage 12 cells (Fig. 2B). To analyze the methylation status of the pluripotency gene promoter, genomic DNA was isolated from the cells. The Oct 4 promoter showed differential methylation patterns between iPSCs and PBMCs detected by bisulfite sequencing. The Oct4 promoter was demethylated in these iPSCs, similar to human ESCs [23] and in contrast to the parental PBMCs (Fig. 2C). Flow cytometry analysis for pluripotency-associated protein expression revealed that they expressed typical human ESC-specific antigens Oct4, Sox2, and SSEA-4 (Fig. 2D). IF analysis confirmed the expression of pluripotency markers Oct4, Sox2, Nanog, and SSEA-4 (Fig. 2E). In order to investigate in vivo differentiation potential, iPSCs were digested, collected, and injected into immunocompromised mice. Around 12 weeks, a visible tumor mass was detected. Histological analysis showed that they formed teratomas consisting of derivatives of all three embryonic germ layers (Fig. 3). Overall, these results suggest that we successfully derived non-integration and feeder-free iPSCs from PBMCs using Sendai viruses carrying Oct4, Sox2, Klf4, and cMyc. The cells express pluripotency markers as confirmed in gene and protein levels. Furthermore, the cells possess multi-lineage differentiation potentials in vivo.

\section{Rock inhibitors promote cell recovery after cell thawing}

Human iPSCs display different passaging properties compared with mouse iPSCs. Unlike mouse iPSCs, they have to be passaged in cell clumps (e.g., using reagents such as dispase, collagenase, or EDTA), instead of single cells. Furthermore, they have very poor cell survival efficiency after cell cryopreservation. The major reason for the low survivability of iPSCs after cell thawing was apoptosis rather than cellular necrosis induced directly by cryoinjury [24]. To increase the cell survival, we tested two Rock inhibitors, RVC and Y-27632, and observed their influence on cell survival post-thaw. In the control group (without Rock inhibitors), the cells displayed very poor survival efficiency, and most cells were dead (Fig. 4A). The cells grew and expanded slowly and only reached 8.69\% confluency after 5 days culturing (Fig. 4B $\& C)$. As expected, both Rock inhibitors dramatically enhanced cell survival efficiency, and RVC 


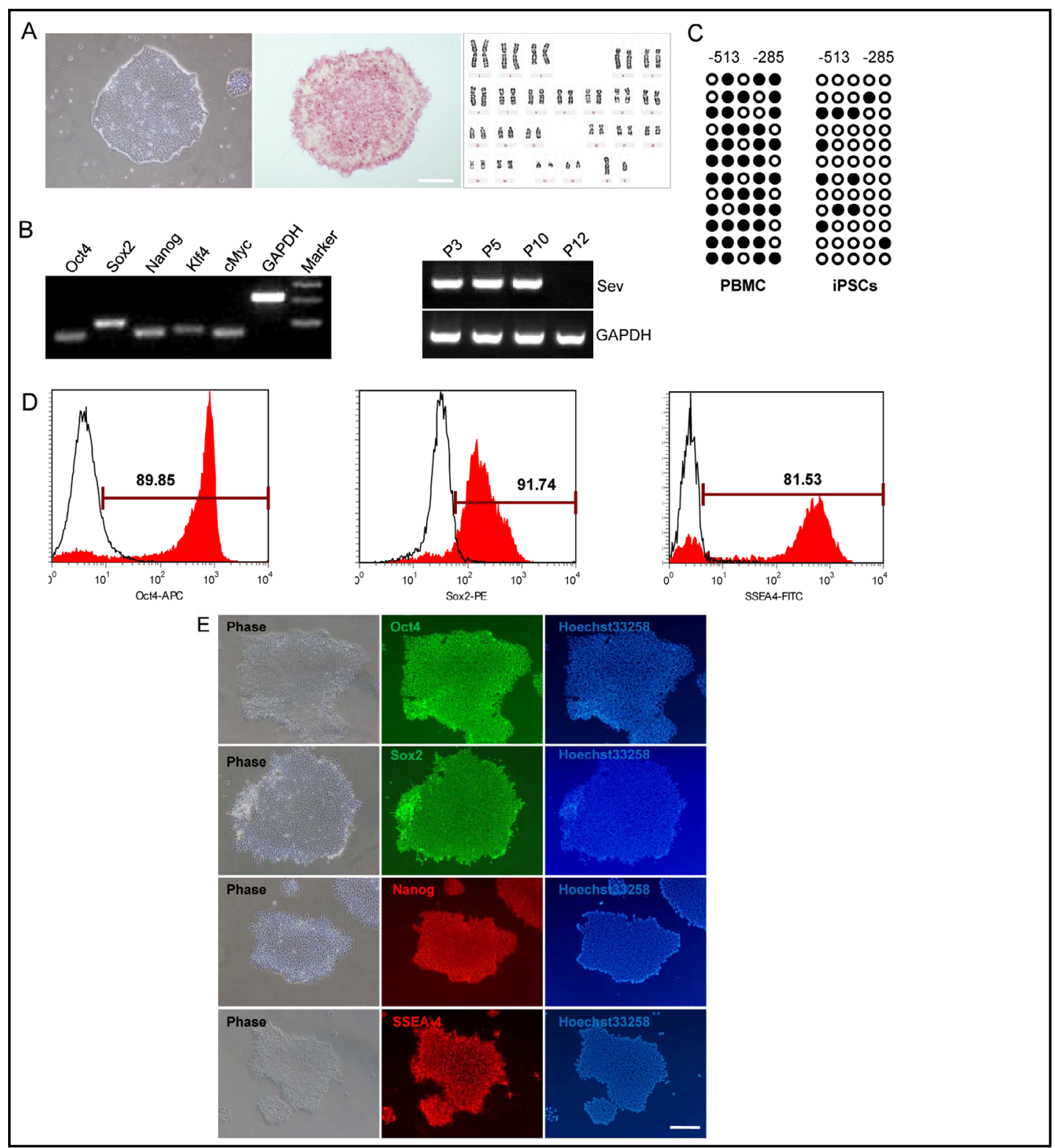

Fig. 2. Characterization of iPSCs. A. Morphology assessment, AP staining, and karyotyping of iPSCs. iPSCs at passage (P) 3 displayed typical human ESC morphology, flat and compact colonies with clear boundaries, high nucleus/cytoplasm ratios, and prominent nucleoli (left panel). These colonies were positive for alkaline phosphatase, displaying a red or purple stain (middle panel). A representative karyogram of an iPSC clone is shown on the right panel. This iPSC clone maintained a normal karyotype (46, XY) after 20 passages of expansion. Bar is $200 \mu \mathrm{m}$. B. Expression of pluripotency and Sendai virus genes by RT-PCR. iPSCs expressed pluripotency genes Oct4, Sox2, Nanog, Klf4, and cMyc (left panel). Sendai virus (Sev) was retained in iPSCs up to P10. After incubating the cells at $38^{\circ} \mathrm{C}$ for 5 days, Sendai virus was completely absent in P12 cells (right panel). C. Analysis of Oct4 promoter DNA methylation status by bisulfite sequencing. The Oct4 promoter of iPSCs was less methylated than that of the parental PBMCs. Methylated CpG sites within the Oct4 promoter are designated by filled circles and unmethylated $\mathrm{CpG}$ sites by unfilled circles. D. Flow cytometric analysis. iPSCs were analyzed for expression of pluripotency markers Oct4, Sox2, and SSEA-4. Unfilled histograms represent matched antibody isotype controls and filled histograms represent expression of markers on the tested cells. E. IF analysis of pluripotency markers in iPSCs. iPSCs were stained in situ by anti-Oct4 (1:100), anti-Sox2 (1:100), anti-Nanog (1:100), and anti-SSEA-4 (1:50) antibodies. Nuclei were highlighted with Hoechst 33258 staining. Bar is $200 \mu \mathrm{m}$.

\section{KARGER}




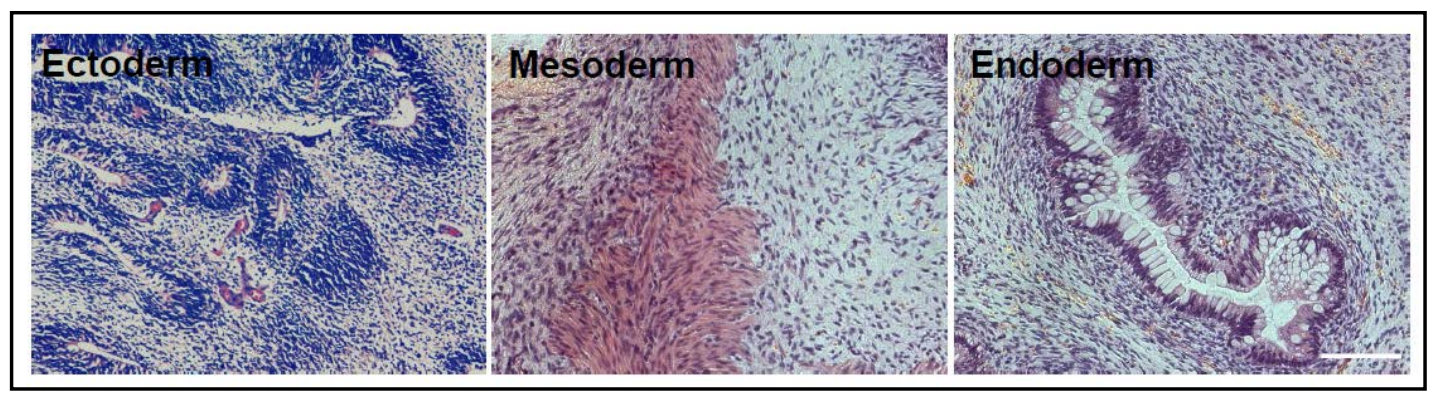

Fig. 3. Teratoma formation. $3 \times 10^{6}$ cells/mouse were injected subcutaneously into the dorsal flank of immunocompromised mice ( $n=6 /$ group). Around 12 weeks, a visible tumor mass was detected. The teratomas were dissected and processed with hematoxylin and eosin staining. The teratomas consisted of derivatives of ectoderm, mesoderm, and endoderm. Bar is $100 \mu \mathrm{m}$.

Fig. 4. Rock inhibitors promote cell recovery after cell thawing. A \& B. Analysis of cell morphology and recovery post-thaw. iPSCs (cell clumps) were thawed and plated on vitronectin-coated plates in E8 medium supplemented with either $10 \mu \mathrm{M}$ Y-27632 (middle panel) or 1X RVC (right panel). Following a 24-hour recovery, iPSCs were fed with E8 medium alone for the remainder of the culture. The control group (left panel) was maintained in E8 medium alone for the entire experiment. Bar is $200 \mu \mathrm{m}$. C. Analysis of cell proliferation after cell thawing. Undifferentiated cell masks were calculated to determine the percentage change in confluency of the cells over time. Data are mean \pm SD of triplicate experiments. * $\mathrm{p}<0.05 ;{ }^{* *} \mathrm{p}<0.01 ;{ }^{* * *} \mathrm{P}<0.001$.

was more effective on cell recovery post-thaw than Y-27632. After 5 days culturing, the cells reached $84.19 \%$ and $34.21 \%$ confluency in the RVC and Y-27632 groups, respectively (Fig. 4B\&C). In all, the results indicate that Rock inhibitors can significantly promote cell recovery and increase the cell survival.

\section{Single cell passaging achieved by Rock inhibitors}

iPSCs are fundamental tools for basic research and applied applications, including cell therapy, drug discovery, and toxicological evaluation. Generally human iPSCs need to be passaged in cell clumps because breaking down the clumps into single cells may

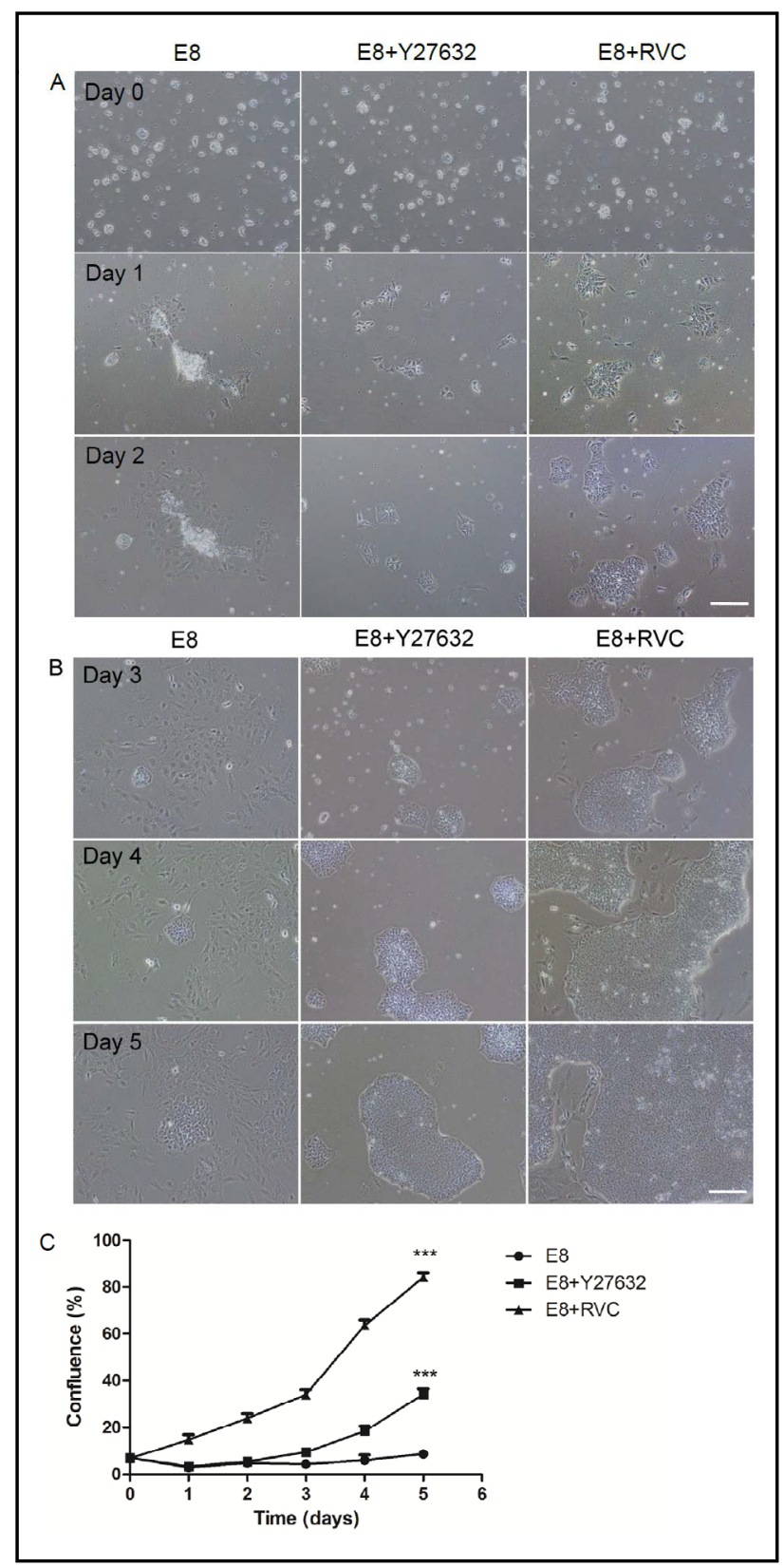

\section{KARGER}




\section{Cellular Physiology

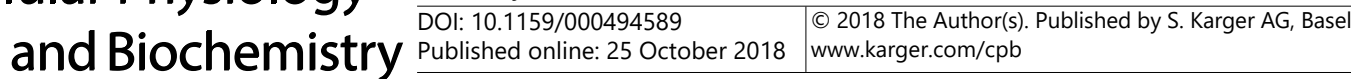 \\ Ye et al.: Peripheral Blood Cells-Derived Non-Integration and Feeder-Free iPSCs}

substantially decrease the cell survival. However, employing clump passaging approach has often been incompatible with downstream experimental applications, such as highthroughput screening, gene editing, and directed lineage differentiation. Thus, we sought to establish an applicable single cell passaging approach for human iPSCs. The major cause for the vulnerability of iPSCs was apoptosis or anoikis (detachment-induced apoptosis) upon cellular detachment and dissociation. They underwent massive cell death particularly after complete dissociation [2528]. Therefore, we investigated whether Rock inhibitors could effectively support single cell passaging. The results showed that addition of either RVC or Y-27632 for 24 hrs post-split could significantly increase the cell survival when the cells were passaged in single cells (Fig. 5A). They showed comparable effectiveness on cell recovery (Fig. 5B). After passaging, the cells grew and expanded rapidly, and maintained the normal iPSC colonies morphology. The colony formation efficiency was markedly increased. Altogether, single cell passaging of human iPSCs could be achieved by addition of Rock inhibitors.

\section{Discussion}

The advent of the iPSC

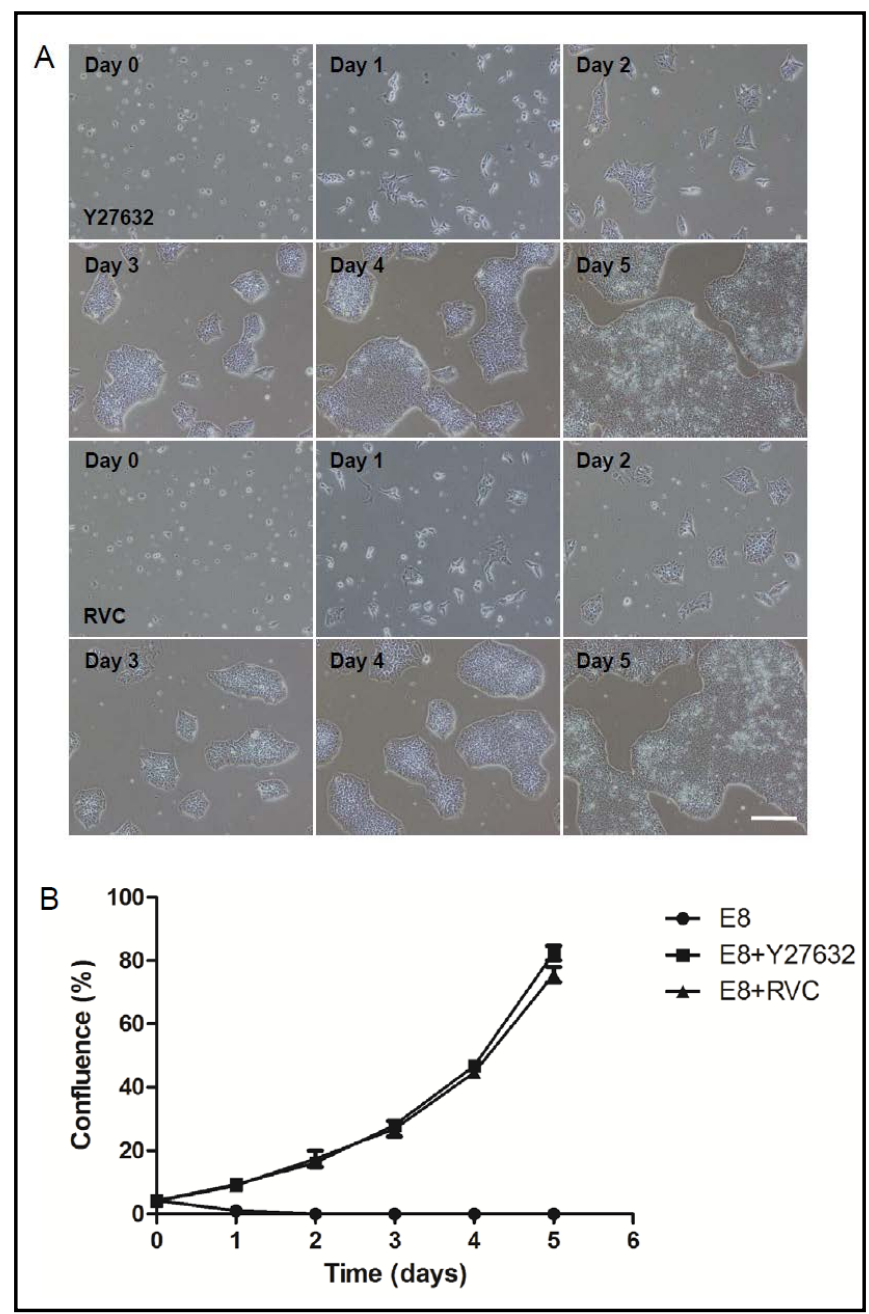

Fig. 5. Single cell passaging achieved by Rock inhibitors. A. Morphology assessment of iPSCs. The cells were dissociated into single cells and plated at a density of 10,000 cells $/ \mathrm{cm}^{2}$ on vitronectin-coated plates in E8 medium supplemented with either $10 \mu \mathrm{M}$ Y-27632 or 1X RVC. Following a 24-hour recovery, iPSCs were fed with E8 medium alone for the remainder of the culture. Bar is $200 \mu \mathrm{m}$. B. Kinetic growth of single iPSCs. Undifferentiated cell masks were calculated to determine the percentage change in confluency of the cells over time. Data are mean \pm SD of triplicate experiments. ${ }^{*} \mathrm{p}<0.05$; ${ }^{* *} \mathrm{p}<0.01$; ${ }^{* * *}$ $\mathrm{P}<0.001$.

technique is a recent breakthrough of stem cell research and application. iPSCs are selfrenewable and have multi-lineage differentiation potentials, similar to ESCs. The development of cellular reprogramming techniques overcomes the two major issues hindering fulfillment of the great potential of human ESCs [1-3]. One is the ethical issue associated with the human ESCs derivation, and the other is the immunological incompatibility induced by histocompatibility-antigenic factors. iPSCs hold great promise for regenerative medicine, disease modeling, and drug development. Thus, there is a need to develop an efficient method to enable the routine derivation of footprint-free and xeno-free iPSCs from many human donor samples, and eventually the derivation of clinical-grade human iPSCs. 


\section{Cellular Physiology Cell Physiol Biochem 2018;50:1318-1331

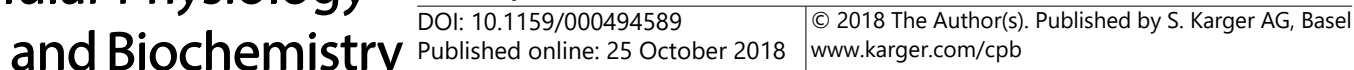 \\ Ye et al.: Peripheral Blood Cells-Derived Non-Integration and Feeder-Free iPSCs}

Although fibroblasts from different sources were initially employed in many studies for the derivation of iPSCs, PBMCs have been widely accepted as a more convenient and almost unlimited resource for cell reprogramming [6, 29-31]. Here, we routinely employed $5 \mathrm{ml}$ peripheral blood for PBMCs isolation and derivation of iPSCs. To make this method easily accessible and minimally invasive, we attempt to cut down the initial volume of peripheral blood. In general, $1 \times 10^{6}$ PBMCs could be efficiently purified from $1 \mathrm{ml}$ peripheral blood. Freshly purified PBMCs from as little as $500 \mu \mathrm{l}$ of peripheral blood were enough for the successful derivation of iPSCs without addition of any reagent for enhancing reprogramming (data not shown). We also found that freshly purified PBMCs cultured for 4-6 days enhanced the cell reprogramming efficiency, consistent with the previous report [32]. Soares, et al. reported generation of hiPSCs from PBMCs using Sendai virus. They first isolated PBMCs from blood and cultured them for 9 days to allow expansion of the erythroblast population before the initiation of cell reprogramming. Then the PBMCs were successfully reprogrammed into iPSCs in the presence of mouse embryonic fibroblasts [33]. Our work further optimized the previous protocol. Before cell reprogramming, there was no need to expand the erythroblasts from PBMCs, and only 4 days culturing were required for cell recovery after cell thawing. Generally, 3 X $10^{5}$ live cells were enough for the successful reprogramming, which is less than the amount ( $5 \times 10^{5}$ live cells) used in the previous protocol [33]. Of note, we could efficiently derive iPSCs under feeder-free conditions, which is favorable for future clinical applications.

Sendai virus reprogramming is an efficient and robust approach with a complete clearance of viral sequences at higher cell passages. The new generation of CytoTune $®$-iPSC 2.0 showed less cytoxocity and higher reprogramming efficiency compared to CytoTune $囚$-iPSC 1.0. However, the cost of the reprogramming kit is very expensive. It was reported that one kit might apply to $24-48$ blood samples (20,000 cells each). The viral activity was maintained close to $80 \%$ activity of its original stock solution after 1 freeze and thaw cycle, and $>50 \%$ activity even after 3 cycles [34]. This allows for thawing Sendai virus and preserving it in small aliquoted frozen stocks, which can greatly decrease the cost of reprogramming. In the current study, we obtained a similar result that efficient reprogramming of PBMCs could be achieved by the virus after 1 freeze and thaw cycle preservation.

Generation of feeder-free iPSCs from PBMCs is technically demanding due to the low reprogramming efficiency under feeder-free conditions. To generate feeder-free iPSCs, we determined whether three types of extracellular substrates could efficiently support cell reprogramming. Geltrex is a reduced growth factor basement membrane extract from murine Engelbreth-Holm-Swarm tumor used routinely for attachment and maintenance of hESCs. The major components of Geltrex include laminin, collagen IV, entactin, and heparin sulfate proteoglycan which provide the foundation for three-dimensional culture studies. Vitronectin and rhLaminin-521 are two recombinant human proteins. Vitronectin is a truncated recombinant protein, corresponding to the amino acid fragment $62-478$ of human vitronectin used as a substrate for the feeder-free culture of human pluripotent stem cells (PSCs). rhLaminin-521 is a recombinant protein that provides a defined surface for feeder-free culture of PSCs. Laminin-521 is a natural component of the stem cell niche in vivo. rhLaminin-521 recapitulates a natural environment for maintenance of self-renewal, normal morphology, pluripotency, and karyotype of PSCs. In the current study, we employed Geltrex, vitronectin, and rhLaminin-521 to replace feeder cells for cell reprogramming. The results revealed that only Geltrex and rhLaminin-521 could efficiently support the initial cell reprogramming process, but vitronectin failed due to loss of attachment between the colonies and vitronectin substrate during the initial reprogramming stage. PBMCs are nonadherent cells, and therefore, tight adherence of PBMCs to the culture well or substrate is particularly important for successful reprogramming. In addition, although the vitronectin failed to support the initial reprogramming, the passaged iPSCs could grow normally and expand on vitronectin substrate, which is consistent with the published report [35]. Previously a similar study reported generation of hiPSCs from PBMCs under feeder-free conditions using Sendai virus [36]. They successfully derived iPSCs using Matrigel matrix in chemically defined conditions, however, Matrigel contained xeno-components. Matrigel

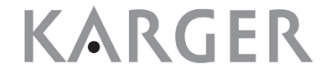




\section{Cellular Physiology Cell Physiol Biochem 2018;50:1318-1331 \begin{tabular}{ll|l} 
DOl: 10.1159/000494589 & O 2018 The Author(s). Published by S. Karger AG, Basel \\
www.karger.com/cpb
\end{tabular} \\ Ye et al.: Peripheral Blood Cells-Derived Non-Integration and Feeder-Free iPSCs}

and Geltrex are both extracted from murine tumor, and use of animal-derived products in derivation, maintenance, and differentiation of iPSCs risks zoonotic disease transmission and immune rejection when transplanted into humans. To avoid potential incorporation of xenogenic products, human substrate proteins are highly desirable. Our study identified that rhLaminin-521 was an ideal substrate for the routine derivation of xeno-free iPSCs.

Efficient cryopreservation and recovery of iPSCs is the first step for the basic research and applications. iPSCs have extremely poor survival rates after cell cryopreservation due to cell apoptosis [24]. In the current study, we found that the iPSCs displayed very poor survival efficiency after thawing with E8 medium alone, and the surviving cells were easy to differentiate. Very few cells could maintain their pluripotency. The reasons for this might be cell apoptosis or some unknown mechanisms, and this is an interesting question worth further investigation. To improve cell recovery, two Rock inhibitors were tested. Both RVC and Y-27632 dramatically enhanced the survival rates post-thaw, and RVC displayed a higher survival rate than that of Y-27632, as demonstrated by the confluency after cell culturing. In addition, unlike Y-27632, there was no requirement for pretreating iPSCs with RVC before cryopreservation [37, 38]. RVC contains a Rock inhibitor that is more selective than traditional Rock inhibitor Y-27632 and is coupled with small molecules including antioxidant and free-radical scavenger properties. This cocktail minimizes apoptosis and necrosis over the first $24 \mathrm{hrs}$ after cell thawing and the off-target pathways affected. Those may be the major reasons for the higher survival rate under the condition of RVC and E8 medium combination. Single cell culture is a standardized and accepted practice in stem cell culture research. By employing Rock inhibitors, we successfully established a single cell passaging approach. Both RVC and Y-27632 could significantly improve the cell survival after single cell passaging while retaining normal cell growth kinetics, morphology, and pluripotency.

\section{Conclusion}

In the present study, we have successfully derived non-integration and feeder-free human iPSCs from peripheral blood cells. Of note, we established effective strategies for efficient cell recovery and single cell passaging, which play pivotal roles on iPSC applications. This study will pave the way to the derivation of clinical-grade human iPSCs for future clinical applications.

\section{Acknowledgements}

This project was supported by the National Science and Technology Support Program (2015BA108B03, H. Y.) and the Innovation Research Foundation of Beijing Institute of Biotechnology (Q. W.). Author Contributions are as follows: Q.W. conceived the project. H. Y. and Q. W. conducted the experiments. Q. W. analyzed the data, and wrote the manuscript.

\section{Disclosure Statement}

The authors declare no conflicts of interest.

\section{References}

1 Takahashi K, Yamanaka S: Induction of pluripotent stem cells from mouse embryonic and adult fibroblast cultures by defined factors. Cell 2006;126:663-676.

2 Takahashi K, Tanabe K, Ohnuki M, Narita M, Ichisaka T, Tomoda K, Yamanaka S: Induction of pluripotent stem cells from adult human fibroblasts by defined factors. Cell 2007;131:861-872. 


\section{Cellular Physiology Cell Physiol Biochem 2018;50:1318-1331 and Biochemistry \begin{tabular}{l|l} 
DOI: 10.1159/000494589 & (c) 2018 The Author(s). Published by S. Karger AG, Basel \\
www.karger.com/cpb
\end{tabular}

3 Yu J, Vodyanik MA, Smuga-Otto K, Antosiewicz-Bourget J, Frane JL, Tian S, Nie J, Jonsdottir GA, Ruotti V, Stewart R, Slukvin, II, Thomson JA: Induced pluripotent stem cell lines derived from human somatic cells. Science 2007;318:1917-1920.

4 Esteban MA, Xu J, Yang J, Peng M, Qin D, Li W, Jiang Z, Chen J, Deng K, Zhong M, Cai J, Lai L, Pei D: Generation of induced pluripotent stem cell lines from Tibetan miniature pig. J Biol Chem 2009;284:17634-17640.

5 Liu H, Zhu F, Yong J, Zhang P, Hou P, Li H, Jiang W, Cai J, Liu M, Cui K, Qu X, Xiang T, Lu D, Chi X, Gao G, Ji W, Ding M, Deng H: Generation of induced pluripotent stem cells from adult rhesus monkey fibroblasts. Cell Stem Cell 2008;3:587-590.

-6 Loh YH, Agarwal S, Park IH, Urbach A, Huo H, Heffner GC, Kim K, Miller JD, Ng K, Daley GQ: Generation of induced pluripotent stem cells from human blood. Blood 2009;113:5476-5479.

-7 Shimada H, Nakada A, Hashimoto Y, Shigeno K, Shionoya Y, Nakamura T: Generation of canine induced pluripotent stem cells by retroviral transduction and chemical inhibitors. Mol Reprod Dev 2009;77:2.

8 Sun N, Panetta NJ, Gupta DM, Wilson KD, Lee A, Jia F, Hu S, Cherry AM, Robbins RC, Longaker MT, Wu JC: Feeder-free derivation of induced pluripotent stem cells from adult human adipose stem cells. Proc Natl Acad Sci U S A 2009;106:15720-15725.

-9 Okita K, Ichisaka T, Yamanaka S: Generation of germline-competent induced pluripotent stem cells. Nature 2007;448:313-317.

10 Fusaki N, Ban H, Nishiyama A, Saeki K, Hasegawa M: Efficient induction of transgene-free human pluripotent stem cells using a vector based on Sendai virus, an RNA virus that does not integrate into the host genome. Proc Jpn Acad Ser B Phys Biol Sci 2009;85:348-362.

11 Hou P, Li Y, Zhang X, Liu C, Guan J, Li H, Zhao T, Ye J, Yang W, Liu K, Ge J, Xu J, Zhang Q Zhao Y, Deng $\mathrm{H}$ : Pluripotent stem cells induced from mouse somatic cells by small-molecule compounds. Science 2013;341:651-654.

$>12$ Jia F, Wilson KD, Sun N, Gupta DM, Huang M, Li Z, Panetta NJ, Chen ZY, Robbins RC, Kay MA, Longaker MT, Wu JC: A nonviral minicircle vector for deriving human iPS cells. Nat Methods 2010;7:197-199.

13 Kaji K, Norrby K, Paca A, Mileikovsky M, Mohseni P, Woltjen K: Virus-free induction of pluripotency and subsequent excision of reprogramming factors. Nature 2009;458:771-775.

14 Okita K, Nakagawa M, Hyenjong H, Ichisaka T, Yamanaka S: Generation of mouse induced pluripotent stem cells without viral vectors. Science 2008;322:949-953.

15 Stadtfeld M, Nagaya M, Utikal J, Weir G, Hochedlinger K: Induced pluripotent stem cells generated without viral integration. Science 2008;322:945-949.

16 Woltjen K, Michael IP, Mohseni P, Desai R, Mileikovsky M, Hamalainen R, Cowling R, Wang W, Liu P, Gertsenstein M, Kaji K, Sung HK, Nagy A: piggyBac transposition reprograms fibroblasts to induced pluripotent stem cells. Nature 2009;458:766-770.

17 Zhou H, Wu S, Joo JY, Zhu S, Han DW, Lin T, Trauger S, Bien G, Yao S, Zhu Y, Siuzdak G, Scholer HR, Duan L, Ding S: Generation of induced pluripotent stem cells using recombinant proteins. Cell Stem Cell 2009;4:381-384.

18 Middleton T, Sugden B: Retention of plasmid DNA in mammalian cells is enhanced by binding of the Epstein-Barr virus replication protein EBNA1. J Virol 1994;68:4067-4071.

-19 Warren L, Manos PD, Ahfeldt T, Loh YH, Li H, Lau F, Ebina W, Mandal PK, Smith ZD, Meissner A, Daley GQ, Brack AS, Collins JJ, Cowan C, Schlaeger TM, Rossi DJ: Highly efficient reprogramming to pluripotency and directed differentiation of human cells with synthetic modified mRNA. Cell Stem Cell 2010;7:618-630.

20 Ban H, Nishishita N, Fusaki N, Tabata T, Saeki K, Shikamura M, Takada N, Inoue M, Hasegawa M, Kawamata S, Nishikawa S: Efficient generation of transgene-free human induced pluripotent stem cells (iPSCs) by temperature-sensitive Sendai virus vectors. Proc Natl Acad Sci U S A 2011;108:14234-14239.

21 Seki T, Yuasa S, Oda M, Egashira T, Yae K, Kusumoto D, Nakata H, Tohyama S, Hashimoto H, Kodaira M, Okada Y, Seimiya H, Fusaki N, Hasegawa M, Fukuda K: Generation of induced pluripotent stem cells from human terminally differentiated circulating T cells. Cell Stem Cell 2010;7:11-14.

22 Schlaeger TM, Daheron L, Brickler TR, Entwisle S, Chan K, Cianci A, DeVine A, Ettenger A, Fitzgerald K, Godfrey M, Gupta D, McPherson J, Malwadkar P, Gupta M, Bell B, Doi A, Jung N, Li X, Lynes MS, Brookes E et al.: A comparison of non-integrating reprogramming methods. Nat Biotechnol 2014;33:58-63.

-23 Yu J, Hu K, Smuga-Otto K, Tian S, Stewart R, Slukvin, II, Thomson JA: Human induced pluripotent stem cells free of vector and transgene sequences. Science 2009;324:797-801. 


\section{Cellular Physiology Cell Physiol Biochem 2018;50:1318-1331 \begin{tabular}{ll|l} 
DOl: 10.1159/000494589 & $\begin{array}{l}\text { O 2018 The Author(s). Published by S. Karger AG, Basel } \\
\text { www.karger.com/cpb }\end{array}$
\end{tabular} \\ Ye et al.: Peripheral Blood Cells-Derived Non-Integration and Feeder-Free iPSCs}

-24 Heng BC, Ye CP, Liu H, Toh WS, Rufaihah AJ, Yang Z, Bay BH, Ge Z, Ouyang HW, Lee EH, Cao T: Loss of viability during freeze-thaw of intact and adherent human embryonic stem cells with conventional slow-cooling protocols is predominantly due to apoptosis rather than cellular necrosis. J Biomed Sci 2006;13:433-445.

25 Amit M, Carpenter MK, Inokuma MS, Chiu CP, Harris CP, Waknitz MA, Itskovitz-Eldor J, Thomson JA: Clonally derived human embryonic stem cell lines maintain pluripotency and proliferative potential for prolonged periods of culture. Dev Biol 2000;227:271-278.

-26 Pyle AD, Lock LF, Donovan PJ: Neurotrophins mediate human embryonic stem cell survival. Nat Biotechnol 2006;24:344-350.

-27 Reubinoff BE, Pera MF, Fong CY, Trounson A, Bongso A: Embryonic stem cell lines from human blastocysts: somatic differentiation in vitro. Nat Biotechnol 2000;18:399-404.

-28 Thomson JA, Itskovitz-Eldor J, Shapiro SS, Waknitz MA, Swiergiel JJ, Marshall VS, Jones JM: Embryonic stem cell lines derived from human blastocysts. Science 1998;282:1145-1147.

-29 Loh YH, Hartung O, Li H, Guo C, Sahalie JM, Manos PD, Urbach A, Heffner GC, Grskovic M, Vigneault F, Lensch MW, Park IH, Agarwal S, Church GM, Collins JJ, Irion S, Daley GQ: Reprogramming of T cells from human peripheral blood. Cell Stem Cell 2010;7:15-19.

-30 Staerk J, Dawlaty MM, Gao Q, Maetzel D, Hanna J, Sommer CA, Mostoslavsky G, Jaenisch R: Reprogramming of human peripheral blood cells to induced pluripotent stem cells. Cell Stem Cell 2010;7:20-24.

-31 Ye Z, Zhan H, Mali P, Dowey S, Williams DM, Jang YY, Dang CV, Spivak JL, Moliterno AR, Cheng L: Humaninduced pluripotent stem cells from blood cells of healthy donors and patients with acquired blood disorders. Blood 2009;114:5473-5480.

32 Su RJ, Baylink DJ, Neises A, Kiroyan JB, Meng X, Payne KJ, Tschudy-Seney B, Duan Y, Appleby N, KearnsJonker M, Gridley DS, Wang J, Lau KH, Zhang XB: Efficient generation of integration-free ips cells from human adult peripheral blood using BCL-XL together with Yamanaka factors. PLoS One 2013;8:e64496.

-33 Soares FA, Pedersen RA, Vallier L: Generation of Human Induced Pluripotent Stem Cells from Peripheral Blood Mononuclear Cells Using Sendai Virus. Methods Mol Biol 2016;1357:23-31.

-34 Beers J, Linask KL, Chen JA, Siniscalchi LI, Lin Y, Zheng W, Rao M, Chen G: A cost-effective and efficient reprogramming platform for large-scale production of integration-free human induced pluripotent stem cells in chemically defined culture. Sci Rep 2015;5:11319.

-35 Kaini RR, Shen-Gunther J, Cleland JM, Greene WA, Wang HC: Recombinant Xeno-Free Vitronectin Supports Self-Renewal and Pluripotency in Protein-Induced Pluripotent Stem Cells. Tissue Eng Part C Methods 2015;10.1089/ten.TEC.2015.0180.

-36 Churko JM, Burridge PW, Wu JC: Generation of human iPSCs from human peripheral blood mononuclear cells using non-integrative Sendai virus in chemically defined conditions. Methods Mol Biol 2013;1036:8188.

-37 Watanabe K, Ueno M, Kamiya D, Nishiyama A, Matsumura M, Wataya T, Takahashi JB, Nishikawa S, Muguruma K, Sasai Y: A ROCK inhibitor permits survival of dissociated human embryonic stem cells. Nat Biotechnol 2007;25:681-686.

-38 Li X, Meng G, Krawetz R, Liu S, Rancourt DE: The ROCK inhibitor Y-27632 enhances the survival rate of human embryonic stem cells following cryopreservation. Stem Cells Dev 2008;17:1079-1085. 